\title{
Proyecciones de clima regional marino sobre España
}

\section{Gabriel Jordà1, Melisa Menéndez ${ }^{2}$, Roland Aznar³, Agustín Sánchez-Arcilla ${ }^{4}$}

\author{
${ }^{1}$ Institut Mediterrani d'Estudis Avançats (IMEDEA: Universitat de les Illes Balears - CSIC), España \\ ${ }^{2}$ Instituto de Hidráulica Ambiental, Universidad de Cantabria, España \\ ${ }^{3}$ Puertos del Estado, España \\ ${ }^{4}$ Laboratori d'Enginyeria Marítima, Universitat Politècnica de Catalunya, España
}

\section{Introducción}

Los cambios en el clima marino debido al calentamiento global pueden tener consecuencias dramáticas para un país como España, donde el océano juega un papel clave en la socioeconomía del país. En España, una gran parte de la población vive en zonas costeras o cerca de ellas y la economía nacional depende en gran medida del turismo, la pesca y las actividades de transporte marítimo (Kersting, 2016). El calentamiento global tendría un impacto en el medio ambiente marino de diferentes maneras. El aumento de las temperaturas globales implica un aumento en la temperatura del océano que a su vez implica un aumento del nivel del mar a través de la expansión térmica de la columna de agua. Además, el calentamiento induce mayores tasas de fusión del hielo terrestre, que también contribuyen a aumentar el nivel del mar. Tal aumento se traduciría en un aumento de las inundaciones costeras, la salinización de los acuíferos o en un aumento de los daños asociados a las tormentas marinas (Nicholls y Cazenave, 2010). Otro efecto potencial del calentamiento global es la modificación de los sistemas de circulación atmosférica (por ejemplo, campos de viento o anomalías de presión a nivel del mar), que se traduce en una modificación de la variabilidad del nivel del mar (básicamente a través de mareas meteorológicas y oleaje) debido a forzamientos meteorológicos. Los cambios en la intensidad, dirección o periodo de las olas de viento pueden alterar i) la morfodinámica de las áreas costeras (por ejemplo, erosión de la playa, retroceso de la línea costa) y ii) aumentar los daños provocados por las tormentas marinas (Field, 2012). Finalmente, el calentamiento global también puede inducir cambios en las corrientes oceánicas y la redistribución de la sal y el calor. Las alteraciones de los campos de temperatura y salinidad pueden tener profundos impactos en ciertos ecosistemas marinos a través del aumento de la mortalidad de ciertas especies, el desplazamiento de nichos ecológicos o la llegada de especies invasoras (por ejemplo, Marbà et al., 2015).

Varios trabajos han analizado el impacto potencial del cambio climático en el medio marino español. Por ejemplo, Losada et al. (2014) y Sánchez-Arcilla et al. (2016) analizaron los posibles impactos costeros, Gomis y Álvarez-Fanjul (2016) se enfocaron en las tendencias de las variables atmosféricas y oceánicas de las últimas décadas y el siglo XXI en áreas cercanas a los puertos españoles, y Kersting (2016) compiló los impactos y la vulnerabilidad de los ecosistemas marinos. Sin embargo, todavía se requiere una revisión actualizada de los impactos proyectados del calentamiento global sobre las variables físicas alrededor de las costas españolas. En el último informe de CLIVAR-España (Pérez-Fiz et al., 2013), Vargas-Yáñez et al. revisaron los signos de los cambios observados en el clima marino alrededor de las costas españolas. Sin embargo, no se abordó el tema de las proyecciones marinas regionales porque en ese momento había pocos estudios disponibles. Hoy en día la existencia de varios estudios nos permite dibujar una imagen general de cuál será el impacto del calentamiento global en el medio marino español. Este es el objetivo de este capítulo.

El capítulo está organizado abordando primero la región mediterránea y luego la región atlántica. Esta distinción es requerida por razones físicas. Ambas regiones se comportan de manera muy diferente y los mecanismos que impulsan la evolución del clima marino en cada región son bastante independientes. Por lo tanto, tiene más sentido separar ambas regiones en el análisis. También hay que señalar que, probablemente debido a la singularidad de la cuenca mediterránea, hay muchos más estudios regionales centrados en el Mediterráneo que en el Atlántico nororiental. Aquí hemos intentado compensar este desequilibrio analizando también estudios globales que arrojan algo de luz sobre la evolución del NE atlántico. Para cada sección estudiamos diferentes variables: oleaje, nivel del mar, temperatura y salinidad. El análisis de las corrientes no está incluido debido a la falta de estudios que se centren en esta variable. Finalmente, vale la pena mencionar que en la actualidad existen pocos estudios regionales basados en los escenarios de RCP (Moss et al., 2010) que son los utilizados en el último informe del IPCC (AR5, Collins et al., 2013). La razón es que las proyecciones oceánicas siempre se retrasan con respecto a las simulaciones climáticas globales. Los modelos oceánicos regionales requieren un forzamiento atmosférico de alta resolución (es decir, regionalización de los modelos globales) que a su vez requieren algún tiempo para producirse después de la aparición de las proyecciones climáticas globales. En consecuencia, aquí revisaremos los estudios utilizando los escenarios RCP y también los SRES (es decir, el informe IPCC-AR4). Cabe señalar que el calentamiento global proyectado en los escenarios RCP comparte algunas similitudes con el proyectado en los escenarios SRES, por lo que ambos conjuntos de escenarios se pueden asimilar. 


\section{A. Región mediterránea A.1 Oleaje}

El clima de oleaje en la costa mediterránea española es más suave que en el Atlántico con alturas de ola medias más pequeñas $(1-1,5 \mathrm{~m})$ y periodos más cortos $(5-6 \mathrm{~s})$ y presenta una variabilidad espacial importante. Las variaciones estacionales muestran un comportamiento diferente con respecto al patrón invierno-verano de las costas atlánticas, con un fuerte patrón semestral (Menéndez et al., 2014) definido por alturas máximas de oleaje en primavera y otoño. Esto es debido a la presencia de ciclones intensos que resultan del aumento de las interacciones aire-mar durante ese periodo.

La evolución del clima de oleaje está fuertemente determinada por la evolución futura de las trayectorias de las tempestades. Aunque la mayoría de las proyecciones muestran un aumento de la intensidad del viento al norte de $45^{\circ} \mathrm{N}$ (Donat et al., 2011; Nikulin et al., 2011), la ubicación del desplazamiento hacia los polos está sujeta a debate (Scaife et al., 2012). Además, a pesar de no ser el factor predominante, los ciclones extratropicales también se ven afectados por la concentración de vapor de agua en la atmósfera, lo que aumenta su intensidad, y por los gradientes de temperatura en la superficie del mar (SST), que afecta su posición y actividad (Bengtsson et al., 2006). Debido a estos factores, la mayoría de los estudios coinciden en que habrá una disminución en el número de ciclones en el Mediterráneo. Sin embargo, hay una falta de consenso sobre si el número de ciclones intensos aumentará o disminuirá (véase por ejemplo, Pinto et al., 2007).

Estos cambios también determinan las proyecciones del oleaje. Lionello et al. (2008) han corrido un modelo regional de olas para todo el Mediterráneo bajo los escenarios A2 y B2. Han descubierto que la altura media significativa (Hs) en gran parte del mar Mediterráneo sería menor durante todas las estaciones a fines del siglo XXI con una reducción mayor durante el invierno (alrededor de $-20 \mathrm{~cm}$ ) en el escenario A2. Estos cambios son similares, aunque más pequeños y menos significativos, en el escenario B2, excepto durante el invierno en el noroeste del mar Mediterráneo, donde se encontrarían alturas de oleaje mayores que en la actualidad. En cuanto a los eventos extremos, estos autores también han encontrado valores más pequeños en escenarios futuros que en el clima actual. Además, han demostrado que, en general, los cambios de $\mathrm{Hs}$, la velocidad del viento y la circulación atmosférica son consistentes. Casas-Prat y Sierra (2013) han corrido un modelo de oleaje regional del Mediterráneo occidental forzado bajo el escenario A1b por 5 regionalizaciones atmosféricas diferentes. Sus resultados muestran un aumento en los vientos y las olas del noroeste sobre el golfo de León, lo que se traduce en un mayor predominio futuro de los estados de vientos marinos en esa región. Estos autores han encontrado cambios de altura proyectados en torno al $\sim 10 \%$ para condiciones medias, $\sim 20 \%$ para clima extremo (nivel de retorno de 50 años) y pequeños cambios en la frecuencia de aparición de olas desde diferentes direcciones ( 5\%). En sus resultados, los patrones espaciales de cambio son complejos y los resultados de los modelos forzados por diferentes modelos climáticos globales (GCM) no concuerdan. Finalmente, en un trabajo basado en downscaling estadístico, Pérez et al. (2015) proyectó una disminución en Hs (Tm) de $-5 \mathrm{~cm}(-0,1 \mathrm{~s})$ en el escenario RCP8.5, $-3 \mathrm{~cm}(-0,2 \mathrm{~s})$ en el escenario RCP4.5, y ningún cambio en el escenario RCP 2.6. Estos resultados concuerdan con los estudios mencionados anteriormente y apuntan a una disminución mayor de la altura de la ola en escenarios de mayor emisión. Sin embargo, es importante tener en cuenta las grandes incertidumbres asociadas a estas proyecciones.

\section{A.2 Nivel del mar}

Modelar la variabilidad del nivel del mar en el mar Mediterráneo no es sencillo. Por un lado, los GCM no tienen suficiente resolución espacial para reproducir los mecanismos principales que controlan la dinámica regional (Calafat et al., 2012a). Por ejemplo, la redistribución del calor dentro de la cuenca está fuertemente sesgada si se utiliza una resolución demasiado gruesa (Llasses et al., 2016). Esto tiene un fuerte impacto en la fiabilidad de las proyecciones de temperatura en el Mediterráneo y, en consecuencia, en la expansión térmica. Por otro lado, a bajas frecuencias la variabilidad del nivel del mar Mediterráneo está fuertemente influenciada por los cambios en el Atlántico cercano (Calafat et al., 2012b), que generalmente no está incluido en los modelos climáticos regionales (RCM) lo que hace que sea imposible para ellos para estimar las tendencias a largo plazo del nivel total del mar (Calafat et al., 2012a).

Hasta ahora, los estudios regionales sobre las proyecciones del nivel del mar Mediterráneo se han centrado en uno de los componentes de la variabilidad del nivel del mar: el componente estérico (es decir, relacionado con los cambios en la densidad de la columna de agua). Sin embargo, debido a la complejidad de la dinámica de la cuenca, se ha demostrado que las proyecciones basadas únicamente en el componente estérico deben considerarse cuidadosamente (Jordà y Gomis, 2013). Carillo et al. (2012) calcularon la evolución del componente estérico en el Mediterráneo hasta 2050, utilizando dos simulaciones forzadas por el modelo ECHAM5 en el escenario A1b. Sus resultados muestran una expansión térmica de $\sim 5 \mathrm{~cm}$ en 2050 . También concluyeron que las diferencias en la temperatura de las aguas del Atlántico que desembocan en el Mediterráneo tienen poco efecto sobre la evolución térmica de la cuenca. Gualdi et al. (2013) utilizaron un conjunto de cinco modelos regionales de atmósfera-océano acoplados para estimar la evolución del componente estérico hasta 2050 bajo el escenario A1b. Sus resultados mostraron un aumento en ese componente de $\sim 15 \mathrm{~cm}$, aunque debe notarse que esto no es completamente representativo del nivel total del mar ya que los efectos de la salinidad no están bien considerados. Adloff et al. (2015) utilizaron un conjunto de modelos oceánicos regionales para producir proyecciones del componente termostérico y para realizar diferentes experimentos de sensibilidad. Sus resultados proyectan una expansión térmica promedio de la cuenca a fines del siglo XXI que oscila entre +34 y $+49 \mathrm{~cm}$ bajo el escenario A2. Esas discrepancias se deben principalmente a las condiciones prescritas para el forzamiento del Atlántico, por lo tanto, de alguna manera en 
desacuerdo con Carillo et al. (2012), que no encontró una sensibilidad significativa al forzamiento del Atlántico. Además, Adloff et al. (2015) mostraron que se obtuvieron resultados similares forzando el modelo bajo el escenario A1b. Finalmente, Somot et al. (2016) siguió un enfoque diferente que combina información de modelos globales con resultados de modelos regionales para inferir una visión más completa de las futuras proyecciones del mar Mediterráneo. A partir de sus resultados, se puede ver que el nivel del mar Mediterráneo básicamente seguirá los cambios globales del nivel medio del mar debido a la combinación de dos factores. En primer lugar, los cambios de circulación en el Atlántico nororiental se traducirán en un aumento del nivel del mar Mediterráneo mayor que el promedio mundial. En segundo lugar, el efecto de la fusión continental de hielo dará lugar a cambios inferiores a la media mundial. Esos dos cambios se compensarán aproximadamente. Al final, el cambio total del nivel del mar para el Mediterráneo se ha proyectado que oscile entre 40 y $100 \mathrm{~cm}$ (Figura 1a). Además, Somot et al. (2016) mostraron que las diferencias regionales dentro de la cuenca podrían diferir hasta $+15 \mathrm{~cm}$ del promedio de la cuenca (Figura 1b).

En cuanto a los eventos extremos del nivel del mar, Marcos et al. (2011) utilizaron un modelo de marea meteorológica forzado por un modelo atmosférico regional bajo los escenarios B2, A1b y A2 para caracterizar su evolución debido al cambio climático. Sus resultados apuntan hacia una reducción en el número promedio de eventos extremos positivos, mientras que los eventos extremos negativos aumentarán a lo largo del siglo XXI. Tales cambios en la magnitud de los eventos extremos pueden atribuirse en parte a la tendencia negativa del nivel medio del mar inducido atmosféricamente para el futuro (Jordà et al., 2012a), con cambios en invierno de hasta $-8 \mathrm{~cm}$ bajo el escenario A2, aunque en general los resultados indican pequeños cambios en comparación con su magnitud actual (reducción de -10\%). Jordà et al. (2012a) también encontraron que algunos eventos en las simulaciones de escenarios podrían ser especialmente fuertes, lo que sugiere que se pueden esperar menos ciclones, con un aumento en la fuerza para algunos de ellos. Conte y Lionello (2013) realizaron un conjunto de simulaciones de marea meteorológica con siete miembros hasta 2050, forzados bajo el escenario A1b. Encontraron una disminución general de $\sim 5 \%$ en la magnitud de las tormentas positivas con cambios de hasta $-10 \%$ en algunos lugares a lo largo de las costas mediterráneas. Cabe destacar que mostraron grandes diferencias entre simulaciones y que los resultados entre modelos no eran espacialmente coherentes. En un trabajo más reciente, Lionello et al. (2016) encontró resultados más consistentes. En otras palabras, las proyecciones de eventos extremos del nivel del mar en el Mediterráneo son muy sensibles a la elección del forzamiento atmosférico.

\section{A.3 Temperatura y salinidad}

La evolución de las propiedades hidrográficas del Mediterráneo está estrechamente relacionada con la evolución del agua y los flujos de calor a través de la superficie del mar. Las proyecciones de modelos regionales (RCM) para la región mediterránea sugieren una disminución de la pérdida
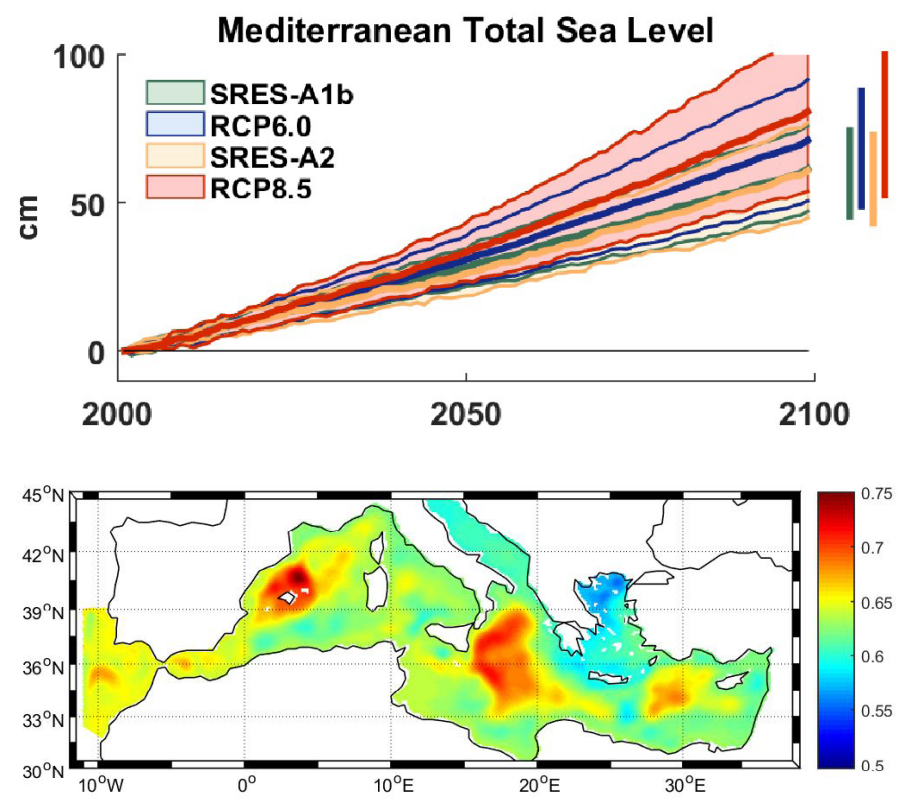

Figura 1. (Arriba), proyecciones del promedio de cuenca del nivel del mar Mediterráneo (en $\mathrm{cm}$, adaptado de Somot et al., 2016). (Abajo), distribución espacial de la proyección de nivel del mar Mediterráneo (en $\mathrm{m}$ ) bajo un escenario moderado (RCP4.5 ० A1b).

de calor en la superficie y un aumento del flujo de agua hacia la atmósfera (Collins et al., 2013). Sánchez-Gómez et al. (2009) analizaron los resultados de 12 RCM forzados por $6 \mathrm{GCM}$ diferentes bajo el escenario A1b. Sus resultados muestran que es probable que el balance hídrico del Mediterráneo se modifique significativamente a fines del siglo XXI. Específicamente, las proyecciones regionales muestran un aumento del $12 \%$ en la evaporación, una reducción del $16 \%$ en la precipitación, una reducción del $24 \%$ en la escorrentía de los ríos y una reducción del $40 \%$ en la contribución del mar Negro. Todos estos cambios resultan en un aumento del $40 \%$ de las pérdidas de agua. La respuesta de las variables hidrológicas al calentamiento global comienza a ser estadísticamente significativa después de 2050, aunque ya se observaron algunas alteraciones antes de 2050. Dubois et al. (2012), utilizando un pequeño conjunto de modelos climáticos regionales acoplados atmósfera-océano (AORCM), mostraron resultados similares. Además, esas simulaciones proyectan un aumento de la pérdida de calor en la superficie para el periodo 2020-2050 que varía de $-1,8$ a $-5,5 \mathrm{~W} / \mathrm{m}^{2}$.

Esos cambios en los flujos superficiales tienen un impacto directo en la evolución de la temperatura y la salinidad en el Mediterráneo (Figura 2). Los GCM corridos bajo el escenario $\mathrm{A} 1 \mathrm{~B}$ proyectan un aumento de la temperatura media de $2,8^{\circ} \mathrm{C}$ a fines del siglo XXI, con una dispersión de $1,0{ }^{\circ} \mathrm{C}$ (Jordà et al., 2012b). Otros estudios basados únicamente en RCM proporcionan resultados muy similares. Gualdi et al. (2013) utilizando un conjunto de cinco AORCM mostraron una proyección de SST promediada para 2050 que oscila entre $1,2^{\circ} \mathrm{C}$ y $2{ }^{\circ} \mathrm{C}$. Adloff et al. (2015), en un conjunto de RCM oceánicos forzados, obtuvieron aumentos 
de la SST promedio en la cuenca que variaban de $+1,7$ a $+3,0{ }^{\circ} \mathrm{C}$ durante el periodo $2070-2099$. Con respecto a la salinidad superficial del mar (SSS), Adloff et al. (2015) mostraron anomalías que variaban entre $+0,48$ y $+0,89$ en el periodo 2070-2099. Sus resultados sugieren que la evolución del agua superficial en la zona del Atlántico próximo, que es muy incierta en los GCM, tiene un gran impacto en la evolución de las masas de agua del Mediterráneo. También destacaron que el aumento de la SSS sería altamente heterogéneo con cambios regionales que podrían exceder de 1, principalmente debido a los cambios en la escorrentía de los ríos.

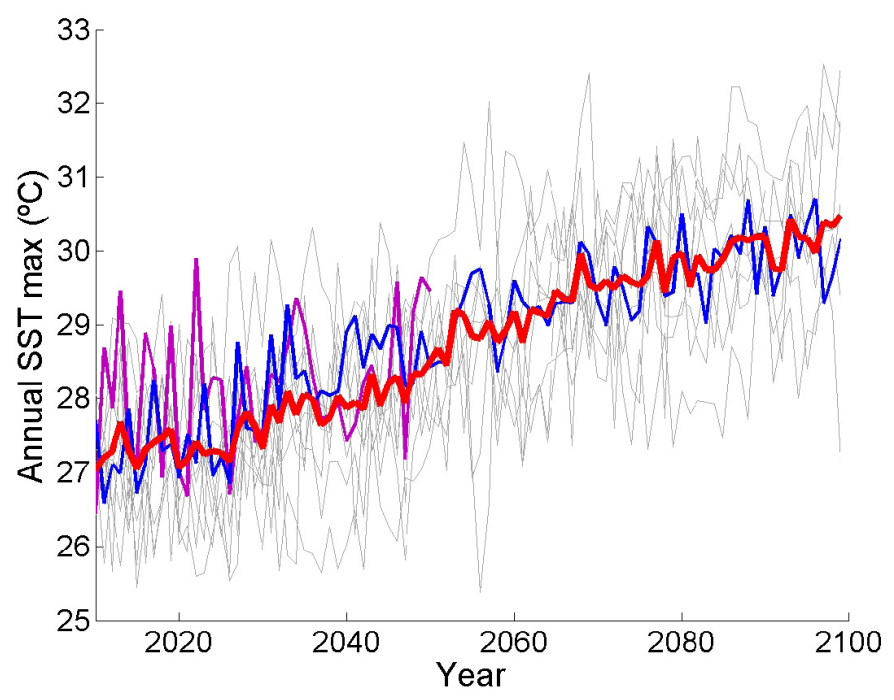

Figura 2. Proyecciones de la temperatura máxima anual de la superficie del mar $\left({ }^{\circ} \mathrm{C}\right)$ para el Mediterráneo occidental a partir de un conjunto de GCM (líneas grises delgadas) y dos RCM oceánicos (líneas violeta y azul). La media del conjunto se muestra en rojo (adaptado de Jordà et al., 2012b).

Los cambios en la temperatura y la salinidad también se sentirían en las capas más profundas. La mayor parte del calentamiento ocurriría en las capas 0-100, 100-600 y 600 -fondo $\left(+0,6{ }^{\circ} \mathrm{C}, 0,5^{\circ} \mathrm{C}\right.$ y $0,4^{\circ} \mathrm{C}$, respectivamente, en 2050), y de manera similar en las cuencas oriental y occidental (Carillo et al., 2012; Adloff et al., 2015). En cuanto a la salinidad, ambos estudios mostraron un aumento de $+0,3-0,5$ en toda la columna de agua durante el mismo periodo. A partir de un análisis de sensibilidad, estos autores han sugerido que la elección de las condiciones de frontera del Atlántico tiene un gran impacto en la evolución de la temperatura y la salinidad de la columna de agua, mientras que la elección del escenario tiene un impacto mucho menor que para las variables de superficie. También mostraron que la penetración de las anomalías de calor y sal desde la superficie a capas profundas varía de acuerdo con la simulación y depende de los cambios en las áreas convectivas, que están influenciadas por el estado histórico de la estratificación vertical y la circulación termohalina mediterránea asociada.

\section{B. Región atlántica \\ B.1. Oleaje}

Las costas del Atlántico español incluyen diferentes áreas regionales: golfo de Vizcaya, mar Cantábrico, golfo de Cádiz y las islas Canarias. En general, la fuerza y la dirección de los vientos del oeste y el recorrido de las tormentas son los principales factores climáticos que determinan el oleaje en el noreste atlántico. Las características del clima eólico marino varían sin embargo significativamente a lo largo de los márgenes atlánticos españoles (Menéndez et al., 2014). Las costas del norte son aquellas que muestran las tempestades de olas más intensas (oleajes energéticos) generadas en toda la cuenca del noreste del Atlántico (Pérez et al., 2014), con alturas promedio de onda de 2-2,5 $\mathrm{m}$ y un periodo de ola medio con valores máximos de $\sim 10 \mathrm{~s}$. En el golfo de Cádiz y las islas Canarias, los efectos del oleaje generado en latitudes altas en el Atlántico Norte son menos intensos (Izaguirre et al., 2010), siendo la altura media de las olas de alrededor de 1-1,5 m y el pico medio de 7-8 s. Además, las características de la onda tienen una fuerte variabilidad temporal, tanto a escalas estacionales como interanuales. Una gran fracción de la energía de las olas a lo largo de la costa atlántica española puede asociarse a patrones de circulación atmosférica de gran escala, principalmente a la Oscilación del Atlántico Norte (NAO), la Oscilación Ártica (AO) y la oscilación del Atlántico oriental (EA) (véase por ejemplo, Izaguirre et al., 2010; Espejo et al.. 2014; Martínez-Asensio et al., 2015).

Con respecto a las proyecciones basadas en regionalizaciones dinámicas del clima de oleaje, el estudio global de Hemer et al. (2013a) es notable ya que proporciona una investigación integrada de 5 estudios independientes realizada en el marco del Proyecto Coordinado del Clima de Oleaje (COWCLIP). Sus resultados sugieren que para el Atlántico nororiental al final del siglo XXI habrá una disminución general de la altura de ola significativa (Hs, 5-10\%) y del periodo medio (Tm) y que esos cambios se concentrarán en la temporada de invierno. Estos resultados están en línea con las conclusiones de otros estudios basados en simulaciones individuales que utilizan el forzamiento de un modelo climático global (por ejemplo, Semedo et al., 2013, Hemer et al., 2013b). Complementariamente hay algunos estudios basados en modelos regionales, que usan una resolución más alta para el forzamiento atmosférico y los modelos de olas. Charles et al. (2012) proyectó una disminución muy similar de la altura de ola en invierno en el golfo de Vizcaya mediante el uso del ARPEGE-Climat GCM en tres escenarios climáticos futuros diferentes (B1, A1B, A2). En particular, mostraron una reducción de $-5 /-10 \%$ en Hs dependiendo de la temporada. Un estudio similar fue realizado por Gomis et al. (2016) usando cuatro modelos climáticos globales parentales (GCM) en el escenario A1b. En todos los casos, los cambios proyectados fueron modestos (menos del $10 \%$ de reducción al final del siglo XXI en todas las variables), y encontraron que la elección del GCM que impulsa el modelo atmosférico regional puede conducir a cambios significativos en los resultados.

Las proyecciones estadísticas proporcionan un complemento interesante a las simulaciones dinámicas del clima de 
a)

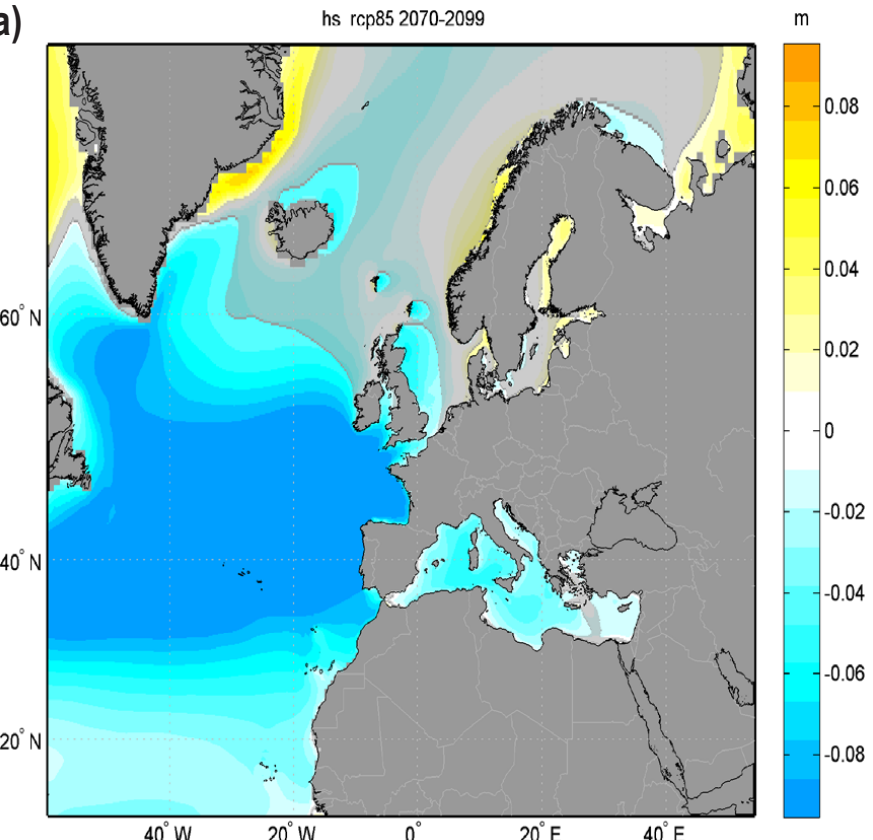

b)

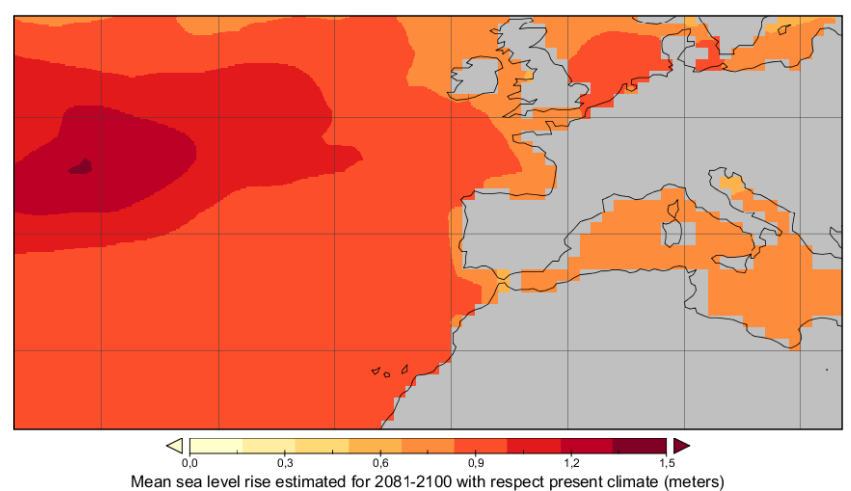

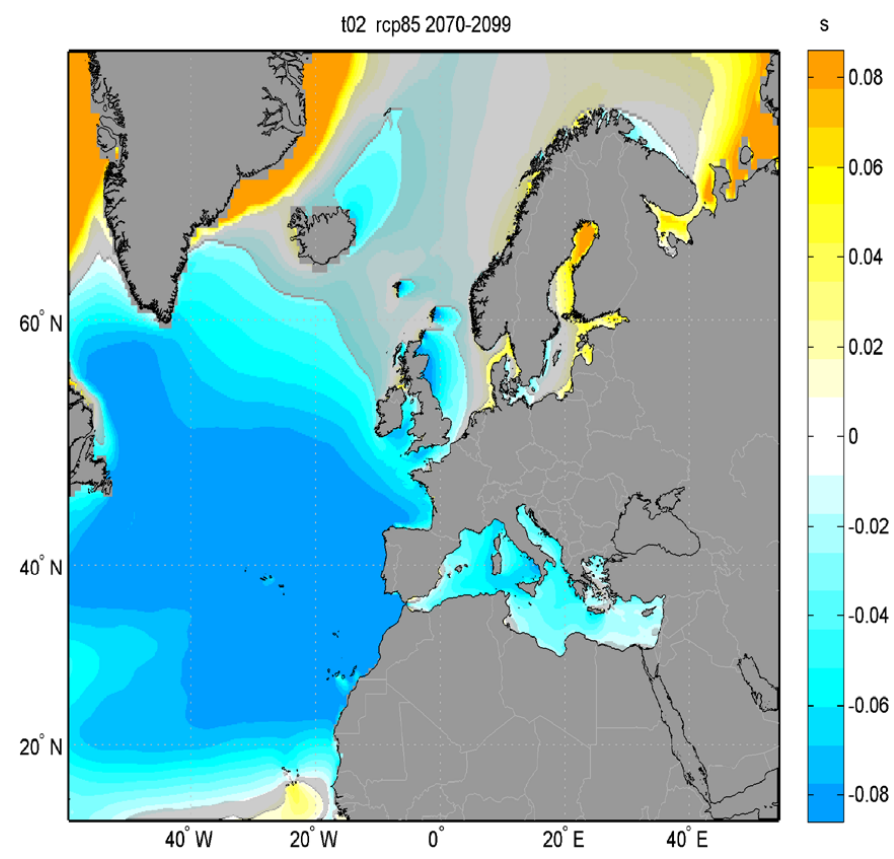

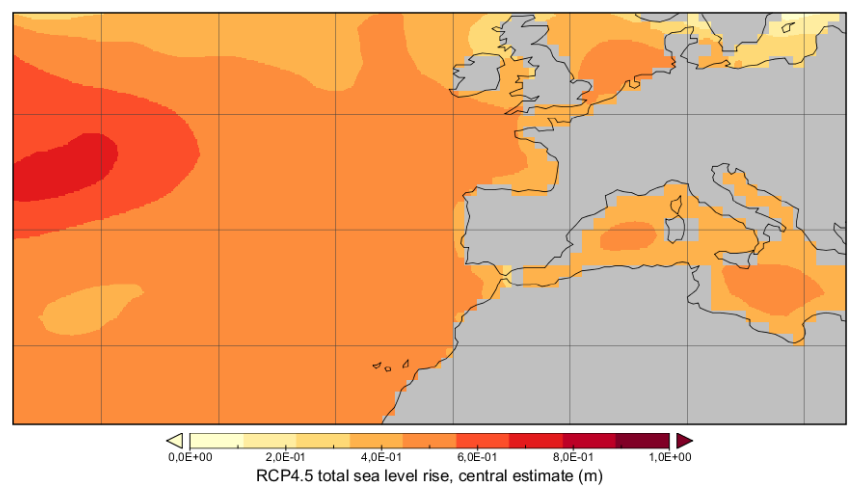

Figura 3. a) Altura significativa de ola (izquierda) y periodo medio (derecha) proyectados en el escenario RCP8.5 para el periodo 2070-2100 y expresados como anomalías con respecto al periodo 1970-2000 (adaptado de Pérez et al., 2015). b) Aumento medio del nivel del mar proyectado en el escenario RCP 8.5 (izquierda) y RCP4.5 (derecha) para el periodo de 20 años 2081-2100 y expresado como anomalías con respecto al periodo 1986-2005 (adaptado de Church et al., 2013).

ola atlántica. Wang et al. (2014) utilizaron el downscaling estadístico basado en la presión del nivel del mar (SLP) como predictor bajo los escenarios RCP4.5 y RCP8.5. Sus resultados sugieren una disminución estadísticamente significativa de $-10 /-15 \mathrm{~cm}$ de $\mathrm{H}$ promedio en el Atlántico NE y una reducción de $-50 /-60 \mathrm{~cm}$ en la altura máxima. Además, no vieron diferencias significativas entre los resultados obtenidos con los nuevos GCM CMIP5 (IPCC-AR5) de lo obtenido con los antiguos GCM CMIP3 (IPCC-AR4). Pérez et al. (2016) desarrollaron un estudio regional de proyección del clima de oleaje multimodelo centrado en la costa europea. Analizaron la calidad de los modelos climáticos sobre el Atlántico Norte (Pérez et al., 2014) y finalmente utilizaron 17 modelos climáticos atmosféricos del CMIP5 para construir el conjunto de simulaciones. Aplicaron un downscaling estadístico basado en la clasificación del tipo de clima utilizando SLP como predictor. Sus resultados sugieren que alrededor de las costas atlánticas ibéricas el cambio proyectado en $\mathrm{Hs}(\mathrm{Tm})$ es $-10 \mathrm{~cm},-5 \mathrm{~cm}$ y $-2 \mathrm{~cm}(-0,08 \mathrm{~s},-0,06 \mathrm{~s}$ y $-0,02 \mathrm{~s})$ para los escenarios
RCP8.5, RCP4. 5 y RCP2.6, respectivamente (véase la Figura 3). Un resultado importante de ese estudio es la conclusión robusta de que los escenarios moderados de emisiones conducen a cambios moderados en la altura de la ola. Sus resultados también muestran que más del $80 \%$ de las simulaciones coinciden en el signo del cambio en las costas españolas. Conclusiones similares han sido obtenidas por Martínez-Asensio et al. (2016).

\subsection{Nivel del mar}

Las proyecciones más actualizadas del nivel del mar medio a escala global (GMSL) basadas en modelos basados en procesos indican que a fines del siglo XXI el GMSL sería significativamente más alta de lo que era a fines del siglo XX. Los resultados sugieren que el aumento probablemente esté en el rango de $0,29-0,55 \mathrm{~m}$ para RCP2.6, 0,36-0,63 m para RCP4.5, 0,37-0,64 m para RCP6.0, y 0,48-0,82 m para RCP8.5 (Church et al., 2013). Las proyecciones basadas en modelos semiempíricos sugieren incluso un aumento mayor, de 1 a 2 m (por ejemplo, Rahmstorf et al., 2007). 

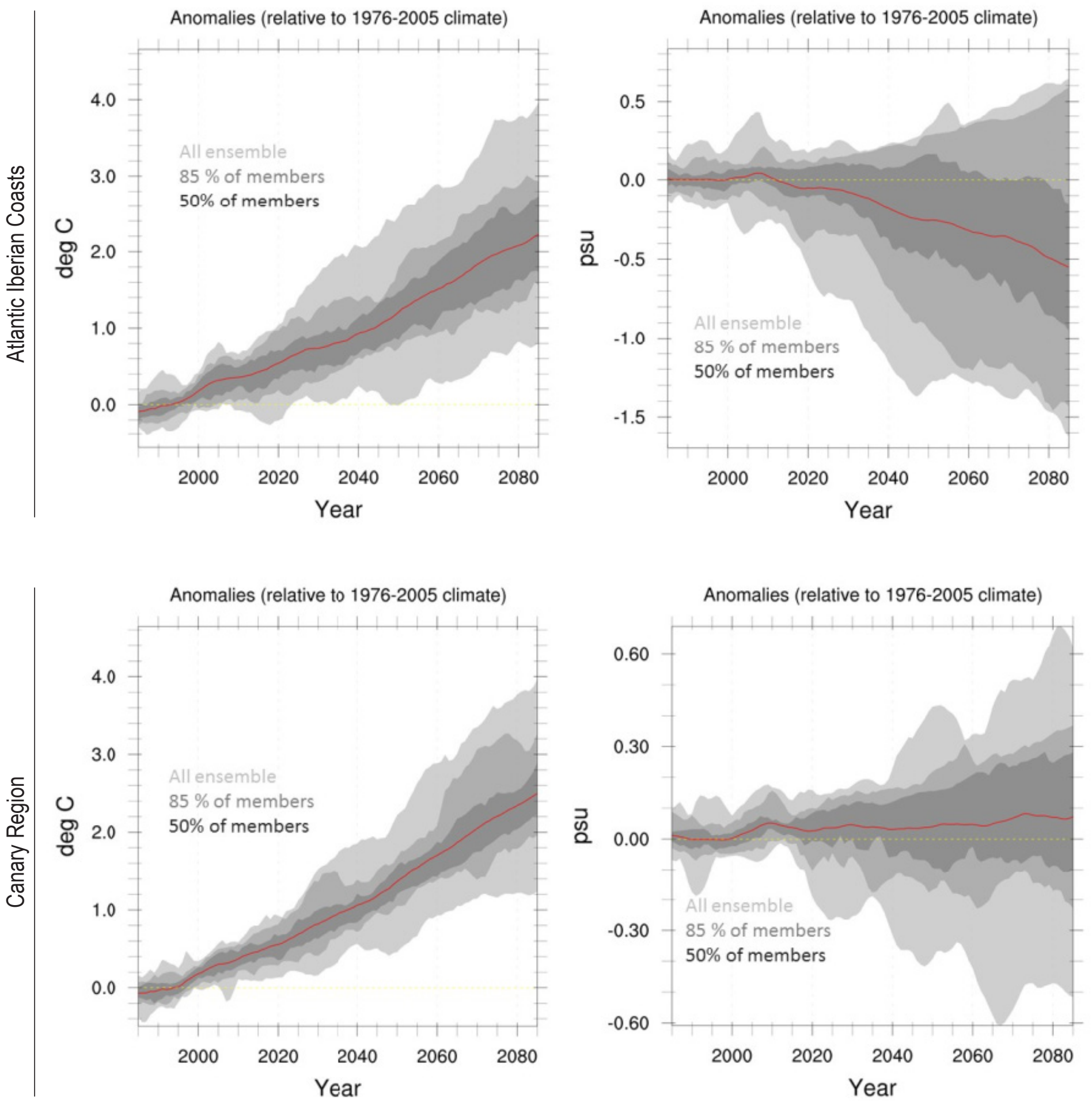

Figura 4. Evolución de la temperatura de la superficie del mar (SST, izquierda) y salinidad de la superficie del mar (SSS, derecha) en el conjunto CMIP5 de GCM bajo el escenario RCP8.5. Los resultados se muestran para la costa atlántica ibérica (arriba) y la región canaria (abajo). La línea roja representa la media del conjunto y los diferentes tonos de gris indican la dispersión del conjunto de simulaciones cuando se considera un $\%$ dado de los miembros. Tenga en cuenta los diferentes ejes verticales en cada figura. 
Sin embargo, es bien sabido que el aumento no sería homogéneo y que se deberían esperar grandes diferencias regionales (por ejemplo, Pardaens et al., 2011). Hasta donde sabemos, solo el estudio de Gomis et al. (2016) ha utilizado un modelo oceánico regional para generar proyecciones del aumento del nivel del mar a lo largo de las costas atlánticas españolas. Trabajaron bajo el escenario A1b, considerando solo los efectos de la expansión térmica y los cambios de circulación y su horizonte temporal fue 2050. Sus resultados sugieren una tasa de nivel del mar de 1,36 $\pm 0,27 \mathrm{~mm} / \mathrm{año}$ y $2,18 \pm 0,54 \mathrm{~mm} /$ año en el $\mathrm{NE}$ atlántico dependiendo del GCM utilizado para proporcionar las condiciones de contorno (ECHAM5 o HADCM3low). Una descripción más completa del aumento del nivel del mar regional ha sido proporcionada por Slangen et al. (2017), que actualiza trabajos anteriores sobre el mismo problema. Han analizado diferentes componentes del aumento del nivel del mar regional utilizando 21 proyecciones del modelo climático CMIP5 con contribuciones regionales basadas en modelos y observaciones de hielo terrestre, aportes de aguas subterráneas y ajuste isostático glacial, incluyendo los efectos gravitacionales debidos a la redistribución de masa. Sus resultados para el Atlántico nororiental en los escenarios RCP4.5/RCP8.5 indican que el nivel total del mar aumentaría $45 / 70 \mathrm{~cm}$ a fines del siglo XXI (Figura $3 b$ ). De ese total, $8 / 12 \mathrm{~cm}$ serían causados por derretimiento del hielo terrestre, $25 / 40 \mathrm{~cm}$ por cambios en la dinámica oceánica, $15 \mathrm{~cm}$ por la contribución de las plataformas de hielo, $5 \mathrm{~cm}$ por almacenamiento de agua terrestre y $2 \mathrm{~cm}$ por GIA.

Un complemento al estudio de la evolución del nivel medio del mar es el análisis de eventos extremos. En particular, los eventos extremos positivos causados por bajas presiones atmosféricas y vientos hacia tierra (es decir, marejadas ciclónicas) que amontonan el agua a lo largo de la costa son los responsables de los mayores daños en las infraestructuras costeras. Marcos et al. (2011) y Jordà et al. (2012a) han analizado esos efectos utilizando un modelo regional de marea meteorológica forzado por el modelo atmosférico regional ARPEGE en los escenarios B2, A1b y A2. Sus resultados muestran que el nivel de retorno de 50 años disminuiría entre 5 y $1 \mathrm{~cm}$ a fines del siglo XXI dependiendo del escenario elegido. Además, se esperan los mayores cambios en el golfo de Vizcaya. Esta reducción está asociada a una disminución en el número de eventos extremos positivos ( 2 eventos/año menos al final del siglo) y a un aumento en la presión atmosférica media en invierno (es decir, el periodo donde ocurren los eventos más extremos) que se traduce en una reducción de $\sim 4 \mathrm{~cm}$ en el golfo de Vizcaya a finales de siglo. Gomis et al. (2016), usaron la misma configuración de modelo pero forzados por otros GCM. Sus resultados no son concluyentes en cuanto las simulaciones forzadas por ECHAM5 y HADCM3low mostraron un cambio ligeramente positivo $(\sim 10 \mathrm{~cm})$ del nivel de retorno de 50 años, mientras que las forzadas por HADCM3red y HADCM3high muestran exactamente lo contrario. Esto sugiere la ausencia de tendencias claras en las tormentas de la región. Es decir, los niveles extremos del mar serán más altos en el siglo XXI debido al aumento en el nivel medio del mar, no debido a un aumento significativo en las tormentas.

\subsection{Temperatura y salinidad}

Existen pocos estudios que analicen la evolución de la temperatura y la salinidad en las costas atlánticas españolas y la mayoría de ellos se basan en los resultados de los GCM (por ejemplo, Chust et al., 2010, Villarino et al., 2015). La Figura 4 muestra la evolución temporal del conjunto de proyecciones CMIP5 SST y SSS para las costas ibéricas del Atlántico y para la región de las islas Canarias. Se proyecta que la SST aumentará alrededor de $2,2{ }^{\circ} \mathrm{C}$ y $2,4{ }^{\circ} \mathrm{C}$ respectivamente a fines de siglo con la mayor tasa de aumento para después de 2050. La diferencia entre ambas regiones se encuentra en la dispersión del conjunto de simulaciones. En las costas ibéricas, el $50 \%$ de los miembros del conjunto proyectan un aumento que oscila entre 1,8 y $2,6^{\circ} \mathrm{C}$, mientras que en la región de Canarias el aumento oscila entre 2,2 y $2,8^{\circ} \mathrm{C}$. Para la SSS, las diferencias entre las regiones son relativamente más grandes. Las proyecciones muestran una bajada de salinidad en el margen ibérico
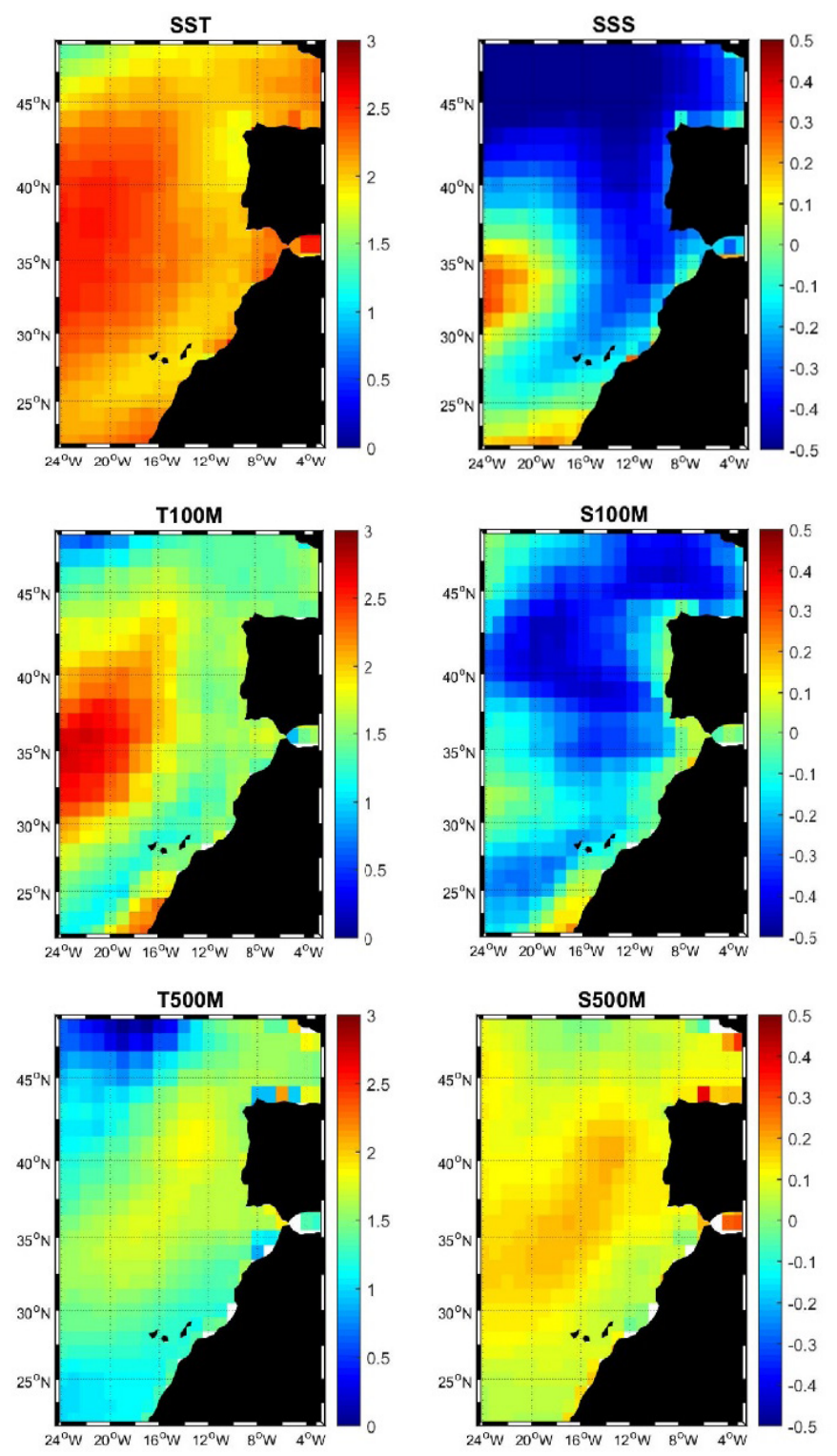

Figura 5. Anomalía media del conjunto de CMIP5 para el periodo (2050-2099) con respecto a (1956-2005). Los mapas se muestran para la temperatura en ${ }^{\circ} \mathrm{C}$ (izquierda) y para la salinidad en psu (derecha), en la superficie (arriba), $100 \mathrm{~m}$ (centro) y $500 \mathrm{~m}$ (abajo). 
y una ligera salinización de la región de Canarias (los cambios proyectados son de $-0,5$ psu y $+0,1 \mathrm{psu}$, respectivamente). Nuevamente, la dispersión del conjunto es mayor para los márgenes ibéricos ( $\mathrm{de}-0,9 \mathrm{a}-0,1 \mathrm{psu})$ que para la región de Canarias (de $-0,1$ a $+0,3$ psu).

Si observamos diferentes profundidades (Figura 5) en el escenario RCP8.5, encontramos un calentamiento en toda la columna de agua. El aumento promedio a lo largo de las costas atlánticas españolas para la segunda mitad del siglo XXI fue de $2-2,5{ }^{\circ} \mathrm{C}, 1-1,5{ }^{\circ} \mathrm{C}$ y $1-1,6{ }^{\circ} \mathrm{C}$ en superficie, $100 \mathrm{~m}$ y $500 \mathrm{~m}$ respectivamente. En cuanto a la salinidad, los resultados son un poco diferentes. Si bien se espera que la salinidad superficial disminuya alrededor de $0,3 \mathrm{psu}, \mathrm{los}$ cambios a $100 \mathrm{~m}$ y $500 \mathrm{~m}$ a lo largo de las costas españolas son apenas significativos. Con respecto a la salinidad del océano abierto se espera que disminuya también a $100 \mathrm{~m}$, mientras que aumentaría a $500 \mathrm{~m}$. Con respecto a la estratificación vertical, los resultados apuntan a un aumento en la estratificación de la columna de agua (temperaturas más altas y salinidades más bajas en las capas superiores), lo que podría tener implicaciones para los ecosistemas costeros.

Los GCM muestran un comportamiento de algún modo distinto a lo largo del margen ibérico en comparación con el mar abierto. Su resolución espacial es demasiado gruesa para ser fiable y, por lo tanto, las proyecciones regionales deberían preferirse a ese análisis al tener una escala más fina. Usando un modelo de circulación regional, Gomis et al. (2016) muestran resultados que son altamente consistentes con la imagen general proporcionada por los GCM. Gracias a la mayor resolución de su modelo, han podido obtener una imagen más compleja sobre los procesos que tendrían influencia sobre la evolución de la temperatura (Figura 6) y la salinidad en la región. En particular, han encontrado que cerca del margen continental, el aumento de la temperatura global podría contrarrestarse mediante un aumento del afloramiento estacional. Cabe destacar que el afloramiento intensificado podría ser lo suficientemente fuerte como para dar lugar a tendencias negativas de temperatura a lo largo de las costas ibéricas, mientras que a lo largo de la costa africana solo daría como resultado una reducción de las tendencias positivas de temperatura. Con respecto a la salinidad, los resultados sugieren que el eventual aumento de salinidad derivado de un afloramiento más intenso no sería suficiente para contrarrestar la bajada de salinidad mencionada anteriormente.

\section{Discusión y conclusiones}

En este capítulo, hemos revisado el conocimiento actual sobre cómo el calentamiento global afectará el medioambiente marino español durante las próximas décadas. En cuanto al clima de oleaje, las proyecciones basadas en modelos numéricos y estadísticos sugieren una pequeña reducción $(<10 \%)$ en la altura de la ola y el periodo medio tanto en las costas españolas del Atlántico como del Mediterráneo.

Con respecto al nivel del mar, se espera que los valores del Mediterráneo y el NE atlántico sigan la tendencia positiva del nivel medio del mar mundial. El resultado global es un aumento significativo del nivel medio del mar a finales del siglo XXI, que oscila entre 45 y $100 \mathrm{~cm}$ en el Mediterráneo y entre 35 y $70 \mathrm{~cm}$ en el Atlántico nororiental, dependiendo del escenario, con una incertidumbre asociada a esas proyecciones de aproximadamente $\pm 40 \mathrm{~cm}$.

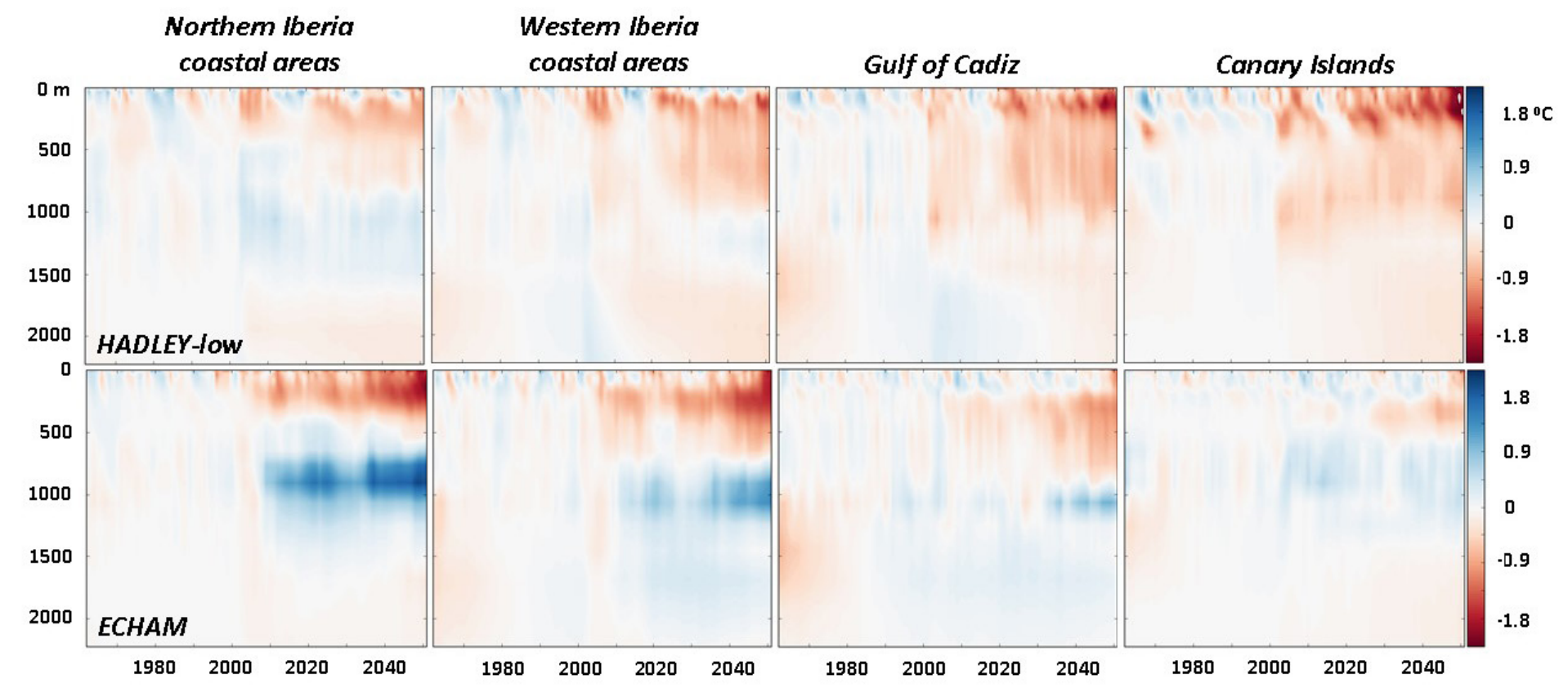

Figura 6. Diagramas de Hovmöller: evolución de los perfiles de anomalías de temperatura (en ${ }^{\circ} \mathrm{C}$, con respecto al promedio de 1971-2000) promediados para las zonas costeras del norte y oeste de la península ibérica, el golfo de Cádiz y las islas Canarias. Los datos corresponden a un RCM oceánico forzado con un escenario A1B de HADLEY-low y ECHAM GCM. 
En cuanto a la temperatura, todos los modelos proyectan un aumento de la temperatura en el Mediterráneo español y las aguas del Atlántico, en consonancia con el aumento previsto en el flujo de calor de superficie. Sin embargo, la magnitud de la desaceleración de la Circulación Meridional Atlántica de Retorno (AMOC), que es incierta, puede modular ese calentamiento en el Atlántico Norte. En particular, una disminución en el transporte de la AMOC implicaría menos calor advectado hacia el NE atlántico. Por lo tanto, dependiendo de las características de la AMOC, deberíamos esperar una tasa de calentamiento diferente a lo largo de las costas ibéricas españolas. Esos efectos también se notarían en la región de Canarias, pero en menor medida. Además, algunos estudios regionales han sugerido que el aumento proyectado en los vientos favorables al afloramiento costero también puede desencadenar un afloramiento más intenso en el margen occidental de la península ibérica. El aumento de la cantidad de aguas afloradas puede (al menos parcialmente) contrarrestar el calentamiento del mar abierto en una banda estrecha a lo largo de la costa occidental ibérica.

En cuanto a la salinidad, el aumento de los flujos de agua dulce en latitudes altas del Atlántico Norte y/o el aumento de la fusión del hielo en Groenlandia traería aguas más dulces hacia las costas del Atlántico Nororiental. Por otro lado, se espera que la pérdida de agua dulce aumente en el Mediterráneo induciendo un aumento en la salinidad de la cuenca, aunque la advección de aguas menos saladas provinientes del Atlántico puede contrarrestar parcialmente este proceso. En cuanto a los eventos extremos, está claro que el impacto de las futuras tormentas marinas aumentará debido al aumento del nivel medio del mar, pero no está claro si el número e intensidad de tormentas cambiará en el futuro. Algunos resultados apuntan a una disminución en el número de tormentas pero también a un aumento de los eventos más intensos, aunque la significación estadística de esos resultados es débil. El último informe del Grupo Intergubernamental de Expertos sobre el Cambio Climático (IPCC) afirma que existe una gran incertidumbre asociada con vientos y tormentas futuras (Collins et al., 2013).

Además de la evolución del régimen medio, para los impactos costeros es muy importante caracterizar la evolución de los eventos extremos. Algunos estudios han abordado proyecciones de eventos extremos de oleaje y de variación del nivel del mar, aunque sus resultados no fueron concluyentes. Los resultados más coherentes que se obtienen a partir de la mayor parte de la generación actual de modelos muestran un desplazamiento hacia el polo de las trayectorias que siguen las tempestades del hemisferio norte para un clima más cálido en el futuro. Sin embargo, ese desplazamiento depende de la superficie polar y del calentamiento de la troposfera tropical superior junto con los diferentes niveles de cambio de la AMOC. Las discrepancias en cómo los GCM proyectan la evolución de esos factores determinan las incertidumbres en los detalles del desplazamiento hacia el polo de la trayectoria de las tempestades.

Un resultado importante de esta revisión es que hemos encontrado relativamente pocos estudios que aborden el cambio climático marino regional en el sur de Europa. Esto es especialmente problemático en el lado atlántico donde el número de estudios es extremadamente limitado y la mayoría de los que están relacionados con la hidrografía y el nivel del mar se basan en modelos climáticos globales. Esto es un serio inconveniente ya que los GCM no fueron diseñados para abordar las particularidades de esa región y a menudo pasan por alto los mecanismos básicos que pueden afectar las proyecciones regionales. En el Mediterráneo, el número de estudios regionales es mayor, pero aún pequeño, lo que impide el análisis preciso de la incertidumbre. Por último, cabe señalar que, en todos los casos, la gran mayoría de los estudios se basan en escenarios de SRES (IPPC AR4), por lo que no se tienen en cuenta los resultados más recientes del IPCC-AR5.

En cuanto a las fuentes de incertidumbre de las proyecciones regionales, el factor más determinante en general es el GCM utilizado para forzar los modelos regionales (atmosféricos y oceánicos). Aunque los GCM pueden estar de acuerdo en la gran escala, pueden ser muy diferentes en los cambios proyectados a pequeña escala y esto a su vez tiene un fuerte impacto en la incertidumbre de las proyecciones regionales. Por ejemplo, la mayoría de los GCM proyectan una disminución del AMOC, pero no están de acuerdo con el monto de su desaceleración. Por lo tanto, la trayectoria de las aguas dulces del Atlántico Norte hacia el $\mathrm{NE}$ atlántico es muy diferente entre los modelos, por lo que es la evolución de la salinidad en las costas españolas ibéricas. De manera similar, la mayoría de los GCM proyectan un desplazamiento hacia el norte de la trayectoria de las tormentas, pero la ubicación exacta es incierta. En consecuencia, las proyecciones del clima de viento sobre el Mediterráneo (y por lo tanto de la marejada ciclónica o el oleaje) dependen en gran medida del GCM elegido para forzar el sistema de modelado regional. En cuanto al escenario de emisión, los resultados apuntan a mostrar cambios más fuertes en los escenarios más pesimistas. Esto está claro para el nivel del mar y la temperatura. Sin embargo, para la salinidad y el oleaje, la relación no es tan robusta. Desafortunadamente, el número de simulaciones regionales es demasiado pequeño para poder producir una estimación precisa del presupuesto de incertidumbre. En este sentido, queremos señalar que los estudios basados en modelos individuales deben considerarse con precaución. Las incertidumbres en las proyecciones son grandes, por lo que las conclusiones basadas en un pequeño número de simulaciones (o incluso en una sola) pueden ser engañosas. Una forma de superar esta limitación es intentar comprender el mecanismo físico detrás de los cambios y analizar su robustez.

Los cambios proyectados en la temperatura y el nivel del mar para las próximas décadas son los resultados más sólidos que hemos encontrado. El aumento de la temperatura en toda la columna de agua y el aumento del nivel del mar en todas las costas españolas es muy probable que suceda a lo largo de este siglo. Los detalles de las heterogeneidades espaciales y el valor exacto de esos aumentos están sujetos a incertidumbres relacionadas con el escenario de emisión, las incertidumbres de modelado y la variabilidad natural. Sin embargo, esas incertidumbres son relativamente pequeñas 
en comparación con el cambio promedio proyectado. Por lo tanto, el aumento previsto de la temperatura y el nivel del mar debe considerarse una amenaza grave. Por lo tanto, existe una necesidad apremiante de comenzar a desarrollar estrategias de adaptación para proteger las infraestructuras costeras y los entornos naturales. Aunque se ha realizado algún trabajo desde una perspectiva teórica hasta nuestro conocimiento, no hay planes específicos para transferir esas ideas de adaptación al mundo real. Esto debería ser abordado por las administraciones lo antes posible.

\section{Agradecimientos}

Este trabajo es una contribución al proyecto CLIFISH (CTM2015-66400-C3-2-R) financiado por el Ministerio de Economía y Competitividad (MINECO). G. Jordà y M. Menéndez agradecen su contrato Ramón y Cajal (RYC2013-14714 y RYC-2014-16469 respectivamente) financiado por MINECO. G. Jordà también quiere expresar su agradecimiento al Gobierno Regional de las Islas Baleares. Los datos de CMIP5 utilizados para producir algunas de las figuras en este capítulo han sido obtenidos del portal web de NOAA Climate Change (http://www.esrl.noaa.gov/ psd/ipcc/). R. Aznar quiere expresar su agradecimiento a la Autoridad Portuaria del Estado español (Puertos del Estado) y a la Agencia Estatal de Meteorología (AEMET).

\section{Referencias}

Adloff, F., Somot, S., Sevault, F., Jordà, G., Aznar, R., Déqué, M., Herrmann, M., Marcos, M., Dubois, C., Padorno, E., Álvarez-Fanjul, E., Gomis, D., 2015: Mediterranean Sea response to climate change in an ensemble of twenty first century scenarios. Clim. Dyn., 45, 2775-2802. doi:10.1007/s00382-015-2507-3.

Bengtsson, L., Hodges, K. I., Roeckner, E., 2006: Storm tracks and climate change. Journal of Climate, 19 (15), 3518-3543.

Calafat, F. M., Jordá, G., Marcos, M., Gomis, D., 2012a: Comparison of Mediterranean sea level variability as given by three baroclinic models. Journal of Geophysical Research: Oceans, 117 (C2).

Calafat, F. M., Chambers, D. P., Tsimplis, M. N., 2012b: Mechanisms of decadal sea level variability in the eastern North Atlantic and the Mediterranean Sea. Journal of Geophysical Research: Oceans, 117 (C9).

Carillo, A., Sannino, G., Artale, V., Ruti, P. M., Calmanti, S., Dell'Aquila, A., 2012: Steric sea level rise over the Mediterranean Sea: present climate and scenario simulations. Clim Dyn., 39 (9-10), 2167-2184. doi:10.1007/s00382-0121369-1.

Casas-Prat, M. y Sierra, J. P., 2013: Projected future wave climate in the NW Mediterranean Sea. Journal of Geophysical Research: Oceans, 118 (7), 3548-3568.

Charles, E., Idier, D., Delecluse, P., Déqué, M., Le Cozannet, G., 2012: Climate change impact on waves in the Bay of Biscay, France. Ocean Dynamics, 62 (6), 831-848.
Church, J. A., Clark, P., Cazenave, A., Gregory, J., Jevrejeva, S., Levermann, A., Merrifield, M., Milne, G., Nerem, R. S., Nunn, P., Payne, A., Pfeffer, W., Stammer, D., Unnikrishnan, A., 2013: Sea level change. En: Climate Change 2013: The Physical Science Basis, editado por Stocker, T. F., Qin, D., Plattner, G.-K., Tignor, M., Allen, S., Boschung, J., Nauels, A., Xia, Y., Bex, V., Midgley, P., Cambridge University Press, Cambridge, UK and New York, NY. USA.

Chust, G., Caballero, A., Marcos, M., Liria, P., Hernández, C., Borja, A., 2010a: Regional scenarios of sea level rise and impacts on Basque (Bay of Biscay) coastal habitats, throughout the 21st century. Estuar Coast Shelf Sci, 87, 113-124.

Collins, M., Knutti, R., Arblaster, J., Dufresne, J.-L., Fichefet, T., Friedlingstein, P., Gao, X., Gutowski, W. J., Johns, T., Krinner, G., Shongwe, M., Tebaldi, C., Weaver, A. J., Wehner, M., 2013: Long-term Climate Change: Projections, Commitments and Irreversibility. En: Climate Change 2013: The Physical Science Basis. Contribution of Working Group I to the Fifth Assessment Report of the Intergovernmental Panel on Climate Change [Stocker, T.F ., Qin, D., Plattner, G.-K., Tignor, M., Allen, S. K., Boschung, J., Nauels, A., Xia, Y., Bex, V., Midgley, P. M. (eds.)]. Cambridge University Press, Cambridge, United Kingdom and New York, NY, USA.

Conte, D. y Lionello, P., 2013: Characteristics of large positive and negative surges in the Mediterranean Sea and their attenuation in future climate scenarios. Global and Planetary Change, 111, 159-173.

Donat, M. G., Leckebusch, G. C., Wild, S., Ulbrich, U., 2011: Future changes in European winter storm losses and extreme wind speeds inferred from GCM and RCM multimodel simulations. Natural Hazards and Earth System Sciences, 11 (5), 1351.

Dubois, C., Somot, S., Calmanti, S., Carillo, A., Déqué, M., Dell'Aquilla, A., Elizalde-Arellano, A., Gualdi, S., Jacob, D., Lheveder, B., Li, L., Oddo, P., Sannino, G., Scoccimarro, E., Sevault, F., 2012: Future projections of the surface heat and water budgets of the Mediterranean sea in an ensemble of coupled atmosphere-ocean regional climate models, Clim. Dyn., 39 (7-8), 1859-1884.

Espejo, A., Camus, P., Losada, I. J., Méndez, F. J., 2014: Spectral ocean wave climate variability based on atmospheric circulation patterns. Journal of Physical Oceanography, 44 (8), 2139-2152.

Fernández Pérez, F., Boscolo, R., Bladé, I., Cacho, I., Castro Díez, Y., Gomis, D., González Sampériz, P., Míguez Macho, G., Rodríguez Fonseca, B., Rodríguez Puebla, C., Sánchez, E., Sotillo, M. G., Valero-Garcés, B., Vargas Yáñez, M., 2010: Clima en España: pasado, presente y futuro. Informe de evaluación del cambio climático regional.

Field, C. B. (ed.), 2012: Managing the risks of extreme events and disasters to advance climate change adaptation: special report of the intergovernmental panel on climate change. Cambridge University Press. 
Gomis, D. y Álvarez-Fanjul, E., 2016: Vulnerabilidad de los puertos españoles ante el cambio climático. Vol. 1: Tendencias de variables físicas oceánicas y atmosféricas durante las últimas décadas y proyecciones para el siglo XXI. Organismo Público Puertos del Estado. Ministerio de Fomento. Madrid, 286 pp.

Gomis, D., Álvarez-Fanjul, E., Jordà, G., Marcos, M., Aznar, R., Rodríguez-Camino, E., Sánchez-Perrino, J. C., Rodríguez-González, J. M., Martínez-Asensio, A., Llasses, J., Pérez, B., Sotillo, M. G., 2016: Regional marine climate scenarios in the NE Atlantic sector close to the Spanish shores. Scientia Marina, 80, 215-234.

Hemer, M. A., Fan, Y., Mori, N., Semedo, A., Wang, X. L., 2013a: Projected changes in wave climate from a multimodel ensemble. Nature climate change, 3 (5), 471-476.

Hemer, M. A., Katzfey, J., Trenham, C. E., 2013b: Global dynamical projections of surface ocean wave climate for a future high greenhouse gas emission scenario. Ocean Modelling, $70,221-245$.

Izaguirre, C., Méndez, F. J., Menéndez, M., Luceño, A., Losada, I. J., 2010: Extreme wave climate variability in southern Europe using satellite data. Journal of Geophysical Research: Oceans, 115 (C4).

Gualdi, S., Somot, S., Li, L., Artale, V., Adani, M., 2013: The CIRCE simulations. Regional Climate Change Projections with Realistic Representation of the Mediterranean Sea. Bulletin of the American Meteorological Society, 94, 65-81.

Jordà, G., Gomis, D., Álvarez-Fanjul, E., Somot, S., 2012a: Atmospheric contribution to Mediterranean and nearby Atlantic sea level variability under different climate change scenarios. Global Planet. Change, 80-81, 198-214.

Jordà, G., Marbà, N., Duarte, C., 2012b: Mediterranean seagrass vulnerable to regional climate warming. Nature Climate Change, 11, vol. 2, 821-824.

Jordà, G. y Gomis, D., 2013: On the interpretation of the steric and mass components of sea level variability. The case of the Mediterranean basin. J. Geophys. Res., 118, 953-963.

Kersting, D. K., 2016: Cambio climático en el medio marino español: impactos, vulnerabilidad y adaptación. Oficina Española de Cambio Climático, Ministerio de Agricultura, Alimentación y Medio Ambiente. Madrid, 166 pp.

Lionello, P., Cogo, S., Galati, M. B., Sanna, A., 2008: The Mediterranean surface wave climate inferred from future scenario simulations. Global and Planetary Change, 63(2), 152-162.

Lionello, P., Conte, D., Marzo, L., Scarascia, L., 2016: The contrasting effect of increasing mean sea level and decreasing storminess on the maximum water level during storms along the coast of the Mediterranean Sea in the mid 21st century. Global and Planetary Change, 151, 80-91.
Llasses, J., Jordà, G., Gomis, D., Adloff, F., Macías, D., Harzallah, A., ..., Sannino, G., 2016: Heat and salt redistribution within the Mediterranean Sea in the Med-CORDEX model ensemble. Climate Dynamics, 1-25.

Losada, I., Izaguirre, C. Díaz, P., 2014: Cambio climático en la costa española. Oficina Española de Cambio Climático, Ministerio de Agricultura, Alimentación y Medio Ambiente. Madrid, $133 \mathrm{pp}$.

Marbà, N., Jordà, G., Agusti, S., Girard, C., Duarte, C. M., 2015: Footprints of climate change on Mediterranean Sea biota. Frontiers in Marine Science, 2, 56.

Marcos, M., Jordà, G., Gomis, D., Pérez, B., 2011: Changes in storm surges in southern Europe from a regional model under climate change scenarios. Glob. Planet. Change, 77, 116-128.

Martínez-Asensio, A., Tsimplis, M. N., Marcos, M., Feng, X., Gomis, D., Jordà, G., Josey, S. A., 2015: Response of the North Atlantic wave climate to atmospheric modes of variability. International Journal of Climatology, 36 (3), 1210-1225.

Martínez-Asensio, A., Marcos, M., Tsimplis, M. N., Jordà, G., Feng, X., Gomis, D., 2016: On the ability of statistical wind-wave models to capture the variability and long-term trends of the North Atlantic winter wave climate. Ocean Modelling, 103, 177-189.

Menéndez, M., Méndez, F. J., Izaguirre, C., Luceño, A., Losada, I. J., 2009: The influence of seasonality on estimating return values of significant wave height. Coastal Engineering, 56 (3), 211-219.

Moss, R., Edmonds, J., Hibbard, K., Manning, M., Steven, K., Rose, S., van Vuuren, D., Carter, T., Emori, S., Kainuma, M., Kram, T., Meehl, G., Mitchell, J., Nakicenovic, N., Riahi, K., Smith, S., Stouffer, R., Thomson, A., Weyant, J. Wilbank, T., 2010: s13 The next generation of scenarios for climate change research and assessment. Nature, 463 (7282), 747.

Nicholls, R. J. y Cazenave, A., 2010: Sea-level rise and its impact on coastal zones. Science, 328 (5985), 1517-1520.

Nikulin, G., Kjellström, E., Hansson, U. L. F., Strandberg, G., Ullerstig, A., 2011: Evaluation and future projections of temperature, precipitation and wind extremes over Europe in an ensemble of regional climate simulations. Tellus A, 63 (1), 41-55.

Pardaens, A. K., Gregory, J. M., Lowe, J. A., 2011: A model study of factors influencing projected changes in regional sea level over the twenty-first century. Climate Dynamics, 36 (9-10), 2015-2033.

Pérez, J., Méndez, F. J., Menéndez, M., Losada, I. J., 2014: ESTELA: a method for evaluating the source and travel time of the wave energy reaching a local area. Ocean Dynamics, 64 (8), 1181-1191. 
Pérez, J., Menéndez, M., Camus, P., Méndez, F. J., Losada, I. J., 2015: Statistical multi-model climate projections of surface ocean waves in Europe. Ocean Modelling, 96, 161-170.

Pinto, J. G., Ulbrich, U., Leckebusch, G. C., Spangehl, T., Reyers, M., Zacharias, S., 2007: Changes in storm track and cyclone activity in three SRES ensemble experiments with the ECHAM5/MPI-OM1 GCM. Climate Dynamics, 29 (2-3), 195-210.

Rahmstorf, S., 2007: A semi-empirical approach to projecting future sea-level rise. Science, 315 (5810), 368-370.

Sánchez-Arcilla, A., García-León, M., Gracia, V., Devoy, R., Stanica, A., Gault, J., 2016: Managing Coastal Environments under Climate Change: Pathways to Adaptation, Science of the Total Environment, doi:10.1016/j.scitotenv.2016.01.124.

Sánchez-Gómez, E., Somot, S., Mariotti, A., 2009: Future changes in the Mediterranean water budget projected by an ensemble of regional climate models. Geophysical Research Letters, 36 (21).

Scaife, A. A., Spangehl, T., Fereday, D. R., Cubasch, U., Langematz, U., Akiyoshi, H., ..., Gettelman, A., 2012: Climate change projections and stratosphere-troposphere interaction. Climate Dynamics, 38 (9-10), 2089-2097.
Semedo, A., Weisse, R., Behrens, A., Sterl, A., Bengtsson, L., Günther, H., 2012: Projection of global wave climate change toward the end of the twenty-first century. Journal of Climate, 26 (21), 8269-8288.

Slangen, A. B. A., Adloff, F., Jevrejeva, S., Leclercq, P. W., Marzeion, B., Wada, Y., Winkelmann, R., 2017: A Review of recent updates of sea-level projections at global and regional scales. Surv. Geophys., 38, 385-406. doi:10.1007/ s10712-016-9374-2.

Somot, S., Jordà, G., Harzallah, A., Darmaraki, S., 2016: The Mediterranean Sea in the future climate projections. En: The Mediterranean Region under Climate Change. A Scientific Update. IRD editions, Marseille. ISBN: 978-27099-2219-7.

Villarino, E., Chust, G., Licandro, P., Butenschön, M., Ibaibarriaga, L., Larrañaga, A., Irigoien, X., 2015: Modelling the future biogeography of North Atlantic zooplankton communities in response to climate change. Mar. Ecol. Prog. Ser., 531, 121-142.

Wang, X. L., Feng, Y., Swail, V. R., 2014: Changes in global ocean wave heights as projected using multimodel CMIP5 simulations. Geophysical Research Letters, 41 (3), 1026-1034. 